\title{
Lean Healthy Children with Short Stature Have Distinct
}

\section{Eating Patterns}

Shir Hadani, ${ }^{1,2}$, Yael Lebenthal ${ }^{1,2}$, Liora Lazar ${ }^{1,2}$, Raanan Shamir ${ }^{2,} 3$, Moshe Phillip ${ }^{1,2}$ and Michal Yackobovitch-Gavan ${ }^{1}$

1. The Jesse Z and Sara Lea Shafer Institute for Endocrinology and Diabetes, National Center for Childhood Diabetes, Schneider Children's Medical Center of Israel, Petah Tikva 49202, Israel

2. Sackler Faculty of Medicine, Tel Aviv University, Tel Aviv 69978, Israel

3. Institute for Gastroenterology, Nutrition, and Liver Diseases, Schneider Children's Medical Center of Israel, Petah Tikva 49202, Israel

\begin{abstract}
Nutrition plays an essential role in normal linear growth in children. Knowledge of the eating styles and dietary consumption of healthy short children from developed countries is scarce. The aim of this study was to investigate the dietary patterns in idiopathic short stature (ISS) and relatively low weight children compared to children with normal stature and weight. This research is a case-control study of 86 pre-pubertal healthy children, mean age $5.9 \pm 1.5$ years. The study group comprised 43 ISS children; 43 age-matched children with normal stature and weight served as controls. Outcome measures included: dietary patterns and physical activity. The absolute daily average energy, protein and carbohydrate intake was significantly lower in the ISS children $(P<0.05)$; after correcting for body surface area, no significant differences were found between groups. Intake of micronutrients calcium, iron, zinc, vitamin A and vitamin C, expressed as percentage from Recommended Dietary Allowance, was significantly lower in the ISS children $(P<0.05)$, who had lower food responsiveness, higher satiety responsiveness, lesser enjoyment of food and were slower eaters $(P<0.001)$. Physical activity was sedentary in both the ISS cases and controls. Our findings in healthy, pre-pubertal, relatively lean ISS children point to a distinct eating pattern with no alteration in physical activity. Understanding the differences in dietary intake and eating behaviors may be beneficial in the development of targeted nutritional intervention for lean ISS children.
\end{abstract}

Key words: Dietary patterns, eating behaviors, lean children, short stature.

\section{Nomenclature}

BMI: $\quad$ Body mass index

BSA: $\quad$ Body surface area

CEBQ: Child eating behavior questionnaire

DRI: Dietary reference intake

EER: Estimated energy requirements

GH: Growth hormone

ISS: Idiopathic short stature

RDA: Recommended dietary allowance

REE: $\quad$ resting energy expenditure

SDS: $\quad$ Standard deviation score

\section{Introduction}

Nutrition plays an essential role in normal linear

Corresponding author: Michal Yackobovitch-Gavan, Ph.D., research field: nutrition. growth and development in children. Proteins, fats and carbohydrates provide the energy and supply the building materials for the newly synthesized tissues, while micronutrients (vitamins and minerals) have regulatory roles in maintaining healthy function of the growing child. During growth the skeleton requires an adequate supply of nutritional factors. Deficiency in energy, protein, iron, vitamin A and zinc can lead to decreased weight gain and stunting of linear growth [1]. To date, most of the studies which explored the role of specific micronutrients in linear growth focused on populations of malnourished children in developing countries [2,3]. Few studies have been carried out in developed countries, where the food supply is not limited [4, 5]. 
Suboptimal dietary intake can be manifested as growth retardation [6]. In affluent societies voluntary food restriction as well as a preference for or avoidance of certain foods may lead to suboptimal caloric intake [7, 8]. Anorexia nervosa, the paradigm of extreme caloric restriction in affluent societies, is commonly associated with impaired growth and if occurring prior to epiphyseal fusion may compromise adult stature [9, 10]. Although voluntary caloric restriction is especially common in adolescent girls it may occur in both genders and throughout childhood; some lean children may develop milder forms of caloric restriction. Indeed, studies focused on children with short stature without an identified cause, who are often lean, found that they were characterized as poor eaters, with a significantly lower weight status than the reference population $[4,5]$. Since such children do not necessarily reveal clinical evidence of malnutrition or deficits in specific micronutrients this may be overlooked by pediatricians and pediatric endocrinologists as the cause of growth retardation [11].

Knowledge of the eating styles and dietary consumption of healthy short children from developed countries is still lacking. The aim of this study was to investigate the dietary patterns in healthy, lean pre-pubertal children with idiopathic short stature (ISS) in comparison to those of children of normal stature and weight.

\section{Materials and Methods}

A case-control study was carried out by the Institute of Endocrinology in Schneider Children's Medical Center of Israel. The study was approved by the local Ethics Committee for Research in Humans of the Israel Ministry of Health. Written informed consent from parents was obtained prior to enrollment in the study for the case group and parental verbal consent was obtained for the control group.

The ISS cases were healthy short and lean children of middle-class families referred to participate in another study aimed to examine the effect of nutritional supplementation on growth in short and lean prepubertal children [12] and fulfilled the inclusion criteria: age of 3-9 years, prepubertal (Tanner 1), with height below 3rd percentile and relatively low weight (weight percentile $\leq$ height percentile) for age and gender according to the 2000 Centers for Disease Control (CDC) growth charts [13]. Controls were healthy children of middle-class families from the community that reside in the geographical vicinity of the ISS cases. Inclusion criteria for controls were age of 3-9 years, prepubertal (Tanner 1), with normal height (between 25th and 95th percentile) and normal body mass index (BMI) for age and gender (between 5th and 85th percentile). Exclusion criteria for both groups were diagnosis of growth hormone $(\mathrm{GH})$ deficiency or treatment with $\mathrm{GH}$, chronic or gastrointestinal disease, genetic syndromes, malignancy, any known organic etiology for slow growth, any chronic medical treatment; also excluded were children for whom both parents were defined as short (height below $2 \mathrm{SD}$ ) and in the control group, children with growth faltering during the previous year (growth faltering was defined as a drop in the height z-score by $>1 \mathrm{SD}$ ) [14]. Calculation of the sample size was based on data of caloric intake in children, presented as calories per $\mathrm{kg}$ body weight [15]. The assumption was that the true difference between the groups is 15 units ( $\mathrm{kcal} / \mathrm{kg}$ body weight) and that the SD of the response variable is 21 , with a $90 \%$ chance of detecting a significant difference at the 2 -sided $5 \%$ level. The aim was to recruit 86 children (43 in each group).

Sociodemographic data, medical history and data on the child's growth, including birth parameters and parental height, were collected from a sociodemographic and clinical questionnaire filled out by the parents. Birth weight was reported as Z-scores using the Fenton 2013 growth calculator for preterm infants [16]. For calculation of mid-parental height, expressed as (SDS): in girls, paternal height minus 13 
$\mathrm{cm}$ was averaged with maternal height; in boys, maternal height plus $13 \mathrm{~cm}$ was averaged with paternal height [17]. Anthropometric assessment included height and weight measurements. Height was measured using a commercial Harpenden stadiometer (Holtain Ltd., Crosswell, United Kingdom). Weight was measured in light clothing using a standard calibrated scale. Height, weight and BMI were expressed as SDS according to the recommendations of the CDC 2000 reference data [13]. Parental short stature was defined as height-SDS $<-2$ SD. Body surface area (BSA) was calculated using the Mosteller formula: BSA $\left(\mathrm{m}^{2}\right)=$ square root of [height $(\mathrm{cm}) \times$ weight $(\mathrm{kg}) / 3,600]$ [18]. Energy and macronutrients intake were standardized to body weight and BSA, whereas micronutrients intake was standardized to Recommended Dietary Allowances (RDA).

Dietary patterns were assessed on the basis of the 3-day food diary and the Child Eating Behavior Questionnaire (CEBQ) [19] filled out by the parents. After brief training by a dietician, the parents were asked to record in a diary, in as great detail as possible, all food and beverages, including portion sizes, consumed over a 3-day period of one week (2 weekdays and 1 weekend), with portion size also described in other terms such as volume or weight. Dietary intake was analyzed using the Tzameret 2 (2009) software developed in collaboration with the Israel CDC and the Ministry of Health. Estimated energy requirements (EER) were calculated for each participant using the Dietary Reference Intake (DRI) equations in accordance with the actual anthropometric parameters and physical activity level as reported by the parents [20]. Parents were asked to describe the amount of moderate and vigorous physical activity engaged in by the child during a regular week. The child's physical activity level was accordingly classified as one of the four DRI physical activity levels (sedentary: typical living activities; low active: typical living activities plus 30-60 minutes of daily moderate activity; active: typical living activities plus at least 60 minutes of daily moderate activity; very active: typical living activities plus at least 60 minutes of daily moderate activity plus an additional 60 minutes of vigorous activity or 120 minutes of moderate activity) [21].

The CEBQ is a validated tool consisting of a 35-item parent-report designed to assess a range of eating styles in children. Parents rate the frequency of their child's behaviors on a 5-point Likert scale (1-never, 2-rarely, 3-sometimes, 4-often, 5-always). The CEBQ has good internal consistency, test-retest reliability, and stability over time, and has been shown to be related to food intake in behavioral tests [19]. Validation of the CEBQ translation from English to Hebrew was done by the research team of the Institute for Endocrinology for this study and other studies (unpublished) in back-translations method. Two reviewers showed decisive agreement in 33 of the 35 items in the questionnaire and high agreement in the remaining 2 items. To conclusively validate the Hebrew version of the questionnaire it was initially administered to the parents of healthy children $(n=34$, mean age 5.8 years, range: 3.0-11.9) from the community - in this sample the Cronbach's alpha values for the questionnaire subscales ranged between 0.818-0.912.

\section{Statistical Analysis}

The data were analyzed using the SPSS software version 20 (SPSS, Inc, Chicago, Illinois). Differences between the two groups in continuous data were examined using independent-sample $t$-tests (normally distributed data) or Mann-Whitney tests (skewed data). The chi-square tests were used to examine the differences in categorical data. Logistic regression analysis was used to explore the relationship between adequate/inadequate growth to nutritional intake and parameters of eating behavior.

\section{Results}

The 46 ISS cases recruited for the study had lower 
height-SDS, weight-SDS and BMI-SDS than the 46 age-matched controls $(P<0.001$, for all parameters, Table 1). Although the mean birth weight-SDS was normal for both ISS cases and controls, the ISS cases had a significantly lower birth weight-SDS than the controls $(-1.36 \pm 0.9$ vs. $-0.18 \pm 0.8, P<0.001)$. No significant differences were found in gestational age at birth (ISS cases: $39.0 \pm 2.4$ vs. controls: $39.3 \pm 2.1, P$ $=0.826$ ). The parents of the ISS cases were significantly shorter than those of the controls (mid-parental height-SDS $-0.98 \pm 0.6$ vs. $0.4 \pm 0.6, P$ $<0.001$ ). In the ISS group, $7 \%$ of the fathers and $16 \%$ of the mothers were defined as having short stature whereas in the control group, none of the parents were short.

The daily average intake of energy (kcal), protein (grams) and carbohydrates (grams) of the ISS cases was significantly lower than that of the controls. After correcting energy, protein and carbohydrate intake according to $\mathrm{kg}$ body weight, the daily average intake of the ISS cases was significantly higher than that of the controls $(P<0.001)$ but after correction according to body size (per BSA unit, taking into account height and weight indices), there was no significant difference between the two groups (Table 2). The distribution of macronutrient intake as percentage of the daily average intake of calories was similar in the two groups (protein: $15.0 \pm 2.8 \%$ vs. $15.6 \pm 2.3 \%, P=$ 0.291 ; carbohydrates: $50.9 \pm 7.0 \%$ vs. $50.4 \pm 4.7 \%, P$
$=0.701$; fat: $34.3 \pm 12.3 \%$ vs. $32.4 \pm 3.5 \%, P=0.756$ ) Moreover, examination of the daily average intake of calories as percentage of the recommended daily intake according to the DRI and EER equations, showed no significant difference between the two groups, both of which met the recommended EER levels $(109.4 \pm 25.7 \%$ vs. $100.7 \pm 19.0 \%, P=0.079)$.

The ISS cases consumed significantly lower levels of iron, zinc, calcium, vitamin A and vitamin C (Fig. 1). For both the ISS cases and controls mean consumption reached the recommended requirements of zinc and vitamin $\mathrm{C}$, but not the RDA recommendation for calcium (both groups consumed on average only $50 \%$ or less). For the ISS cases, mean consumption of iron and vitamin A failed to reach RDA recommendations.

CEBQ scoring [19] showed that the ISS cases were characterized as slower eaters and had a higher sensitivity to satiety, a lower responsiveness to food and lower enjoyment of food, than their controls (Table 3). No significant differences were found in fussiness, emotional under/overeating or drinking. No significant difference was found in the physical activity level of the two groups $(P=0.207)$. For both groups, the mode category of physical activity level was "sedentary".

The logistic regression analysis, which included all the variables that were found to be significant in the univariant analyses (micronutrient intake as percentage

Table 1 Anthropometric characteristics and birth parameters of ISS cases as compared to controls.

\begin{tabular}{llll}
\hline & Cases $(\mathrm{n}=43)$ & Controls $(\mathrm{n}=43)$ & $P$ value \\
\hline Boys/Girls, $\mathrm{n}(\%)$ & $30(69.8) / 13(30.2)$ & $26(60.5) / 17(39.5)$ & 0.365 \\
Age, years & $5.8 \pm 1.4$ & $5.9 \pm 1.6$ & 0.328 \\
Height-SDS & $-2.35 \pm 0.34$ & $0.13 \pm 0.77$ & $<0.001$ \\
Weight-SDS & $-2.82 \pm 0.56$ & $-0.02 \pm 0.65$ & $<0.001$ \\
Body mass index-SDS & $-1.49 \pm 0.71$ & $-0.12 \pm 0.75$ & $<0.001$ \\
Gestational age, weeks & $39.0 \pm 2.4$ & $39.3 \pm 2.1$ & 0.826 \\
Birth weight-SDS & $0.92 \pm-1.36$ & $0.77 \pm-0.18$ & $<0.001$ \\
\hline
\end{tabular}

N, number; SDS, standard deviation score.

Data are presented as mean \pm standard deviation.

Differences between the two groups were tested for statistical significance by using the chi-square test (for gender distribution) and $t$ test (for the rest of variables). 
Table 2 Intake of dietary nutrients: ISS cases vs. controls.

\begin{tabular}{llll}
\hline & Cases $(\mathrm{n}=43)$ & Controls $(\mathrm{n}=43)$ & $P$ value \\
\hline Total daily energy intake, kilocalorie & $1273 \pm 334.5$ & $1484 \pm 343.6$ & 0.005 \\
Kilocalorie/BSA & $1973 \pm 479.2$ & $1823 \pm 338.4$ & 0.097 \\
Kilocalorie/kg body weight & $87 \pm 21.6$ & $14.6 \pm 72$ & $<0.001$ \\
Protein, grams & $47.0 \pm 12.1$ & $57.8 \pm 15.4$ & 0.001 \\
Protein, grams/BSA & $73.2 \pm 18.9$ & $71.0 \pm 16.9$ & 0.578 \\
Protein, grams/kg & $3.2 \pm 0.9$ & $2.8 \pm 0.7$ & 0.014 \\
Carbohydrates, grams & $162.3 \pm 50.7$ & $186.8 \pm 46.1$ & 0.021 \\
Carbohydrates, grams/BSA & $251.9 \pm 74.0$ & $229.8 \pm 49.6$ & 0.107 \\
Carbohydrates, grams/kg & $11.2 \pm 3.3$ & $9.1 \pm 2.1$ & 0.001 \\
Fat, grams & $48.7 \pm 21.4$ & $53.7 \pm 14.8$ & 0.209 \\
Fat, grams/BSA & $74.7 \pm 28.3$ & $65.7 \pm 14.2$ & 0.068 \\
Fat, grams/kg & $3.3 \pm 1.2$ & $2.6 \pm 0.6$ & 0.001 \\
Iron, $m g(\% R D A)$ & $7.7 \pm 2.4(76.9 \pm 24.1)$ & $10.0 \pm 4.0(100.4 \pm 40.1)$ & 0.002 \\
Zinc, $m g(\% R D A)$ & $5.4 \pm 1.4(107.2 \pm 28.4)$ & $6.4 \pm 2.9(128.4 \pm 57.6)$ & 0.034 \\
Calcium, $m g(\% R D A)$ & $419.0 \pm 191.7(41.9 \pm 19.2)$ & $507.5 \pm 209.5(50.8 \pm 21.0)$ & 0.044 \\
Vitamin A, $m c g(\% R D A)$ & $329.1 \pm 228.4(82.3 \pm 57.1)$ & $573.5 \pm 354.3(143.4 \pm 88.6)$ & $<0.001$ \\
Vitamin C, $m g(\% R D A)$ & $68.5 \pm 75.9(274.1 \pm 303.4)$ & $97.7 \pm 69.3(390.7 \pm 277.3)$ & 0.009 \\
\hline
\end{tabular}

Sources: Dietary Reference Intakes: Recommended Dietary Allowances (RDAs) and Adequate Intakes, Vitamins and Minerals, Food and Nutrition Board, Institute of Medicine, National Academies.

Data presented as mean \pm standard deviation.

BSA, Body surface area: BSA $\left(\mathrm{m}^{2}\right)=($ Height $(\mathrm{cm}) \times$ Weight $(\mathrm{kg}) / 3600) \frac{1}{2} ;$; mg-milligrams; meg-micrograms.

Differences between the two groups were tested for statistical significance by using the $t$ test and Mann-Whitney test.

of RDA recommendations and four subcategories of the CEBQ), controlled for age and gender, included the following variables: vitamin A consumption as percentage of $\mathrm{RDA}$ recommendation, $\mathrm{OR}=0.897$ (CI95\% 0.807-0.996); food responsiveness, OR = 0.293 (CI95\% 0.102-0.840), and slowness in eating, $\mathrm{OR}=3.674(\mathrm{CI} 95 \% 1.710-7.893)$. The regression model was significant $\left(\mathrm{R}^{2}=0.468, P<0.001\right)$. According to the regression analysis results, a $10 \%$ increase in the vitamin A intake as percentage of the RDA recommendation might reduce the chance to be in the lean ISS group by $10.3 \%$; being characterized with high responsiveness to food might reduce this by 70.7\%; and being characterized as a slower eater might increase the chance to be in the lean ISS group by 3.67 fold.

\section{Discussion}

Our studied cohort of middle-class, healthy, lean and short pre-pubertal children living in a developed country provided us with the opportunity to explore the eating patterns and dietary intake of children with short stature growing up in an affluent society in which the avoidance of certain foods would be due to the child's choice rather than economic factors. The combination of food diaries together with the eating behavior questionnaires filled out by the parents allowed us to obtain a clearer picture of the children's eating patterns, of both the actual consumption of nutrients and their eating behaviors. We found that although lean ISS children consumed sufficient energy and macronutrients for their body size, they had a lower intake of micronutrients, particularly vitamin A. These lean ISS children had distinct eating patterns: they ate more slowly, reached satiety faster, had a lower responsiveness to food and enjoyed food less than the normal, healthy controls.

The absolute energy intake of the lean ISS children was significantly lower than that of the children with normal height and weight. This finding is in line with previous studies reporting that an inadequate consumption of calories led to impaired linear growth. 


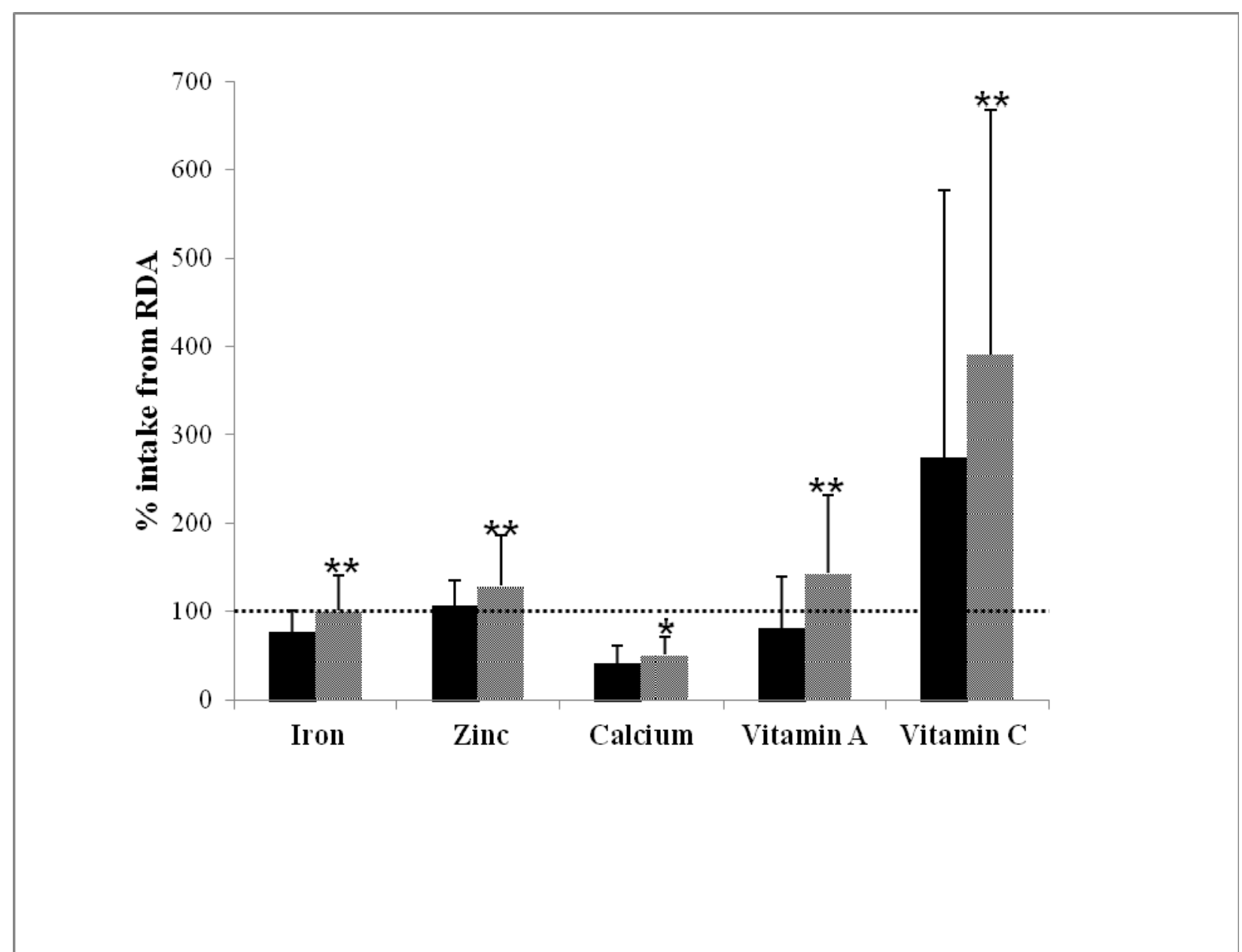

Fig. 1 Micronutrient intake as percentages of Recommended Dietary Allowance (RDA), ISS cases as compared to controls. ISS cases consumed significantly lower levels of iron, zinc, calcium, vitamin A and vitamin C. Differences between the two groups were tested for statistical significance by using the $t$ test and Mann-Whitney test. Solid bars, cases; patterned grey bars, controls.

$* P$ value $<0.05$

$* * P$ value $<0.01$

Table 3 Child eating behavior questionnaire's scores, ISS cases as compared to controls.

\begin{tabular}{llll}
\hline Behavior/Eating style & Cases $(\mathrm{n}=43)$ & Controls $(\mathrm{n}=43)$ & $P$ value \\
\hline Food responsiveness & $1.5 \pm 0.5$ & $1.8 \pm 0.5$ & 0.003 \\
Satiety responsiveness & $3.9 \pm 0.7$ & $3.2 \pm 0.5$ & $<0.001$ \\
Fussiness of food & $3.2 \pm 0.9$ & $2.9 \pm 0.9$ & 0.210 \\
Enjoyment of food & $2.8 \pm 0.9$ & $3.5 \pm 0.7$ & $<0.001$ \\
Slowness in eating & $3.5 \pm 0.8$ & $2.7 \pm 0.7$ & $<0.001$ \\
Emotional overeating & $1.7 \pm 0.7$ & $1.8 \pm 0.6$ & 0.243 \\
\hline
\end{tabular}

Data presented as mean \pm standard deviation.

Each item was rated on a five-point Likert scale that ranges from 1-never to 5-always.

Differences between the two groups were tested for statistical significance by using the $t$ test and Mann-Whitney test.

[1]. Surprisingly, after correcting for body size (BSA), the energy intake of lean and short children was similar to that of their peers and when correcting only for weight, the lean and short children consumed even more calories than their peers. It is of note that in both the lean ISS children and the children with normal height and weight dietary consumption was in accordance with the DRI recommendations for energy 
and macronutrients. Furthermore, no differences were found in the distribution of macronutrient intake from the total calories consumed daily. These findings suggest that the growth impairment in our cohort of children with ISS cannot be explained by insufficient energy and macronutrients intake alone. Another plausible explanation could be increased resting energy expenditure (REE) in lean ISS children, which could lead to an energy balance insufficient to achieve optimal growth. The question arises as to whether there is any difference in REE between the lean ISS children and their peers. A study by Nishimoto et al. [22] found that in ISS children the adjusted REE was significantly higher than in children with normal height and weight, assuming that a higher REE in lean ISS children equates a higher energy need, and furthermore that the actual REE was significantly higher than the age-matched and height-matched basal metabolic rate as calculated according to the DRI equations. This may suggest that the calculated energy intake according to the DRI was not sensitive enough and that there might be an underestimation of the energy level required to account for the energy expenditure, taking into consideration the energy which is required for growth. Since in the present study REE was not measured, we can only assume that according to the information provided by the food diaries and the physical activity questionnaire, our lean ISS children had a relatively higher REE.

Interestingly, lean ISS children consumed significantly lower levels of all the micronutrients (iron, zinc, calcium, vitamin $\mathrm{A}$ and vitamin $\mathrm{C}$ ) than did their peers. These findings are in accordance with those of previous observational and clinical studies showing: (1) a significant correlation between the vitamin A intake level and growth retardation [23, 24]; (2) a relationship between iron-deficiency anemia and impaired physical growth [25, 26]; and (3) conclusive evidence linking the intake of zinc to child growth, showing that zinc supplementation led to a significant improvement in linear growth, especially in children with low zinc status $[3,27,28]$. In contradistinction to our study, all these studies were conducted in developing countries and the study population included malnourished children, so that aside from the lack of these micronutrients there was probably a lack in energy and macronutrients, which possibly contributed to growth retardation. The micronutrient intake may well reflect the quality of the food consumed [29]. It is well established that the more one eats a variety of foods from all food groups, the more likely one is to consume a diverse and sufficient amount of micronutrients. Thus, the relative insufficiency in micronutrient intake in lean ISS children could also reflect the differences in the type and variety of foods consumed by them and by their peers. Yet, these findings should be interpreted with caution since plasma concentrations of micronutrients were not assessed.

The current study found that the lean ISS children have poor eating patterns. In line with the findings of Rosenbloom [4], our lean ISS children attained satiety faster, had lower enjoyment of food and ate more slowly, which might explain the lower absolute amount of calories consumed, and thus the lower intake of other nutrients. Another explanation could be that these lean ISS children consume less diverse food because they have less interest in and enjoyment from food, and this might inadvertently impact their intake of micronutrients. In contrast to the study by Wudy et al. [5], that described ISS children as "picky eaters", our lean ISS children and the children with normal height and weight, scored similarly on the "fussiness of food" subscale.

Genetic factors must also be given some consideration since about 80 percent of the difference in height between people is determined by genetic factors, whereas 20 percent can be attributed to environmental effects, mainly nutrition [30]. Although the parents of the lean ISS children were on average of normal height, they were significantly shorter than the parents of their peers. Lean ISS children would be 
expected to be in the height range of their genetic height, approximately -1 SD (according to mid-parental height-SDS), which was within the normal range. However, the lean ISS children's height SDS, approximately $-2.4 \mathrm{SD}$, was substantially shorter than their target height, which suggests that, apart from genetics, others factors such as nutrition contributed to their short stature. According to logistic regression, possible explanatory variables for the short stature in our studied cohort of lean ISS children were "slow eating", "low responsiveness to food", and lower consumption of vitamin A. Since the issue of causality could not be fully addressed it was not possible to determine whether nutritional insufficiencies and/or poor eating patterns were indeed the cause of their short stature.

\section{Conclusion}

Although in lean ISS children the caloric intake adjusted for body size may be similar to that of their peers, the quality of their diet is suboptimal since their consumption of micronutrients is inadequate. It is plausible that relative deficiency or insufficiency of these micronutrients might have contributed to the reduced height of these children. Yet, we can only assume that the ISS children have a relative insufficiency in micronutrients as compared to their peers, since data on plasma micronutrients level are lacking. Increased awareness of pediatricians and endocrinologists involved in the management of these short children should facilitate nutritional assessment and intervention. Further research is needed to elucidate the optimal nutritional and/or behavioral intervention which might improve the growth of these children.

\section{Acknowledgements}

This work was performed by S. Hadani, MSc in partial fulfillment of the MSc thesis requirements of the Sackler Faculty of Medicine, Tel Aviv University, Tel Aviv, Israel.
The authors declare that they have no competing interests.

\section{References}

[1] Yablonski, G., Yackobovitch-Gavan, M., and Phillip, M. 2009. "Nutrition and Bone Growth in Pediatrics, Endocrinol." Metab. Clin. North. Am. 38: 565-86.

[2] Bhandari, N., Bahl, R., and Taneja, S. 2001. "Effect of Micronutrients Supplementation on Linear Growth of Children.” Br. J. Nutr. 85 (suppl 2): S131-7.

[3] Ramakrishnan, U., Aburto, N., McCabe, G., and Martorell, R. 2004. "Multimicronutrient Interventions but Not Vitamin A or Iron Interventions Alone Improve Child Growth: Results of 3 Meta-Analyses." J. Nutr. 134: 2592-602.

[4] Rosenbloom, A. L. 2009. "Idiopathic Short Stature: Conundrums of Definition and Treatment." Int. J. Pediatr. Endocrinol. 2009: 1-5.

[5] Wudy, S. A., Hagemann, S., Dempfle, A., Ringler, G., Blum, W. F., and Berthold, L. D. 2005. "Children with Idiopathic Short Stature Are Poor Eaters and Have Decreased Body Mass Index." Pediatrics 116: 52-7.

[6] Thibault, H., Souberbielle, J. C., Taieb, C., and Brauner, R. 1993. "Idiopathic Prepubertal Short Stature Is Associated with Low Body Mass Index." Horm. Res. 40: $136-40$.

[7] Cohen, P., Rogol, A. D., Deal, C. L., Saenger, P., Reiter, E. O., and Ross, J. L. 2008. "Consensus Statement on the Diagnosis and Treatment of Children with Idiopathic Short Stature: A Summary of the Growth Hormone Research Society, the Lawson Wilkins Pediatric Endocrine Society, and the European Society for Paediatric Endocrinology Workshop." J. Clin. Endocrinol. Metab. 93: 4210-7.

[8] Lifshitz, F., Friedman, S., Smith, M. M., Cervantes, C., Recker, B., and O'Connor, M. 1991. "Nutritional Dwarfing: A Growth Abnormality Associated with Reduced Erythrocyte $\mathrm{Na}+, \mathrm{K}+$ ATPase Activity." Am. J. Clin. Nutr. 54: 997-1004.

[9] Modan-Moses, D., Yaroslavsky, A., Kochavi, B., Toledano, A., Segev, S., and Balawi, F. 2012. "Linear Growth and Final Height Characteristics in Adolescent Females with Anorexia Nervosa." PLoS One 7: e45504.

[10] Modan-Moses, D., Yaroslavsky, A., Novikov, I., Segev, S., Toledano, A., and Miterany, E. 2003. "Stunting of Growth as a Major Feature of Anorexia Nervosa in Male Adolescents." Pediatrics 111: 270-6.

[11] Cole, C. R., and Lifshitz, F. 2008. "Zinc Nutrition and Growth Retardation." Pediatr. Endocrinol. Rev. 5: 889-96. 
[12] Lebenthal, Y., Yackobovitch-Gavan, M., Lazar, L., Shalitin, S., Tenenbaum, A., and Shamir, R. 2014. "Effect of a Nutritional Supplement on Growth in Short and Lean Prepubertal Children: A Prospective, Randomized, Double-Blind, Placebo-Controlled Study." J. Pediatr. 165: 1190-3.

[13] Kuczmarski, R. J., Ogden, C. L., Grummer-Strawn, L. M., Flegal, K. M., Guo, S. S., and Wei, R. 2000. "2000 CDC Growth Charts: United States." Adv. Data 314: $1-27$.

[14] Grimberg, A., Ramos, M., Grundmeier, R., Feemster, K. A., Pati, S., and Cucchiara, A. J. 2009. "Sex-based Prevalence of Growth Faltering in an Urban Pediatric Population." J. Pediatr. 154: 567-72.

[15] Beal, V. A. 1953. "Nutritional Intake of Children I. Calories, Carbohydrate, Fat and Protein." J. Nutr. 50: 223-34.

[16] Fenton, T. R., and Kim, J. H. 2013. “A Systematic Review and Meta-Analysis to Revise the Fenton Growth Chart for Preterm Infants." BMC Pediatr. 13 (1): 59 .

[17] Nwosu, B. U., and Lee, M. M. 2008. "Evaluation of Short and Tall Stature in Children." Am. Fam. Physician 78: 597-604.

[18] Feber, J., and Krásnicanová, H. 2012. "Measures of Body Surface Area in Children." In Handbook of Anthropometry, edited by Preedy, V. R. New York: Springer, 1249-56.

[19] Wardle, J., Guthrie, C., Sanderson, S., and Rapoport, L. 2001. "Development of the Children's Eating Behavior Questionnaire." J. Child. Psychol. Psychiatry 42: 963-70.

[20] Institute of Medicine of the National Academies, Food and Nutrition Board. 2002. Dietary Reference Intakes: for Energy, Carbohydrate, Fiber, Fat, Fatty Acids, Cholesterol, Protein, and Amino Acids. Washington, DC: The National Academies Press.
[21] Otten, J. J., Hellwig, J. P., and Meyers, L. D., eds. 2006. Dietary Reference Intakes, DRI: The Essential Guide to Nutrient Requirements. Washington, DC: The National Academies Press, 84.

[22] Nishimoto, Y., Ida, S., Etani, Y., and Miyatani, S. 2012. "Resting Energy Expenditure in Short Stature Children.” Endocr. J. 59: 265-71.

[23] .Kurugol, Z., Egemen, A., Keskinoglu, P., Darcan, S., and Aksit. S. 2000. "Vitamin A Deficiency in Healthy Children Aged 6-59 Months in Izmir Province of Turkey." Paediatr. Perinat. Epidemiol. 14: 64-9.

[24] Fawzi, W. W., Herrera, M. G., Willett, W. C., Nestel, P., Amin, A., and Mohamed, K. A. 1997. "Dietary Vitamin A Intake in Relation to Child Growth." Epidemiology 8: 402-7.

[25] Owen, G. M., Lubin, A. H., and Garry, P. J. 1971. "Preschool Children in the United States: Who Has Iron Deficiency?" J. Pediatr. 79: 563-8.

[26] Rao, K. V., Radhiah, G., and Raju, S. V. 1980. "Association of Growth Status and Prevalence of Anemia in Preschool Children, Indian." J. Med. Res. 71: 237-46.

[27] Hakimi, S. M., Hashemi, F., Valaeei, N., Seyed-Masood, K., Velayati, A. A., and Boloursaz, M. R. 2006. "The Effect of Supplemental Zinc on the Height and Weight Percentiles of Children." Arch. Iran. Med. 9: 148-52.

[28] Gibson, R. S., Manger, M. S., Krittaphol, W., Pongcharoen, T., Gowachirapant, S., Bailey, K. B., and Winichgoon, P. 2007. "Does Zinc Deficiency Play a Role in Stunting among Primary School Children in NE Thailand?" Br. J. Nutr. 97: 167-75.

[29] Wirt, A., and Collins, C. E. 2009. "Diet Quality-What Is It and Does It Matter?" Public Health Nutr. 12: 2473-92.

[30] Silventoinen. K. 2003. "Determinants of Variation in Adult Body Height.” J. Biosoc. Sci. 35: 263-85. 